\title{
Simulation of Bouncing Solid Particle in Oil: Spherical vs. Polyhedral Particle Shape Effect
}

\author{
Fatemeh Razavi ${ }^{* *}$, Alexandra Komrakova ${ }^{1}$, Carlos F. Lange ${ }^{1}$ \\ ${ }^{1}$ Department of Mechanical Engineering, University of Alberta, Edmonton, Canada \\ *razavi1@ualberta.ca
}

\begin{abstract}
To prepare for the simulation of sand filtration in heavy oil, the detailed flow of individual particles in oil is studied. Investigation of the motion of a bouncing solid particle toward the wall in a fluid helps understand the particle-fluid and particlewall interactions in the numerical model. The numerical results get verified against experimental data through reporting Root Mean Square Error (RMSE). The particle distance-from-wall is measured. Although some numerical research has been carried out on spherical bouncing particle, there have been few numerical investigations into the effect of the non-spherical particles on the trajectory/distance-from-wall of the falling/bouncing particle toward the wall in a fluid. Accordingly, the specific aims of this work are:
\end{abstract}

1) Checking that the developed numerical model can simulate the motion of a bouncing spherical particle. It will result in validating/benchmarking the developed model from the perspective of particle-wall and particle-fluid interactions.

2) Comparative study on the effect of the solid particle spherical shape and polyhedral shape on the trajectory/distance-from-wall of the particle.

Considering the quiescent fluid of silicon oil in a tank made of glass, a steel sphere is falling in oil toward the bottom glass wall. The mass density of the steel sphere is $\rho_{p}=7800 \mathrm{~kg} / \mathrm{m}^{3}$. The Young's modulus of elasticity $E$ is $214 \times 109 \mathrm{~Pa}$ and the Poisson's ratio $v$ is 0.3 . The mass density of silicon oil is $\rho_{f}=970 \mathrm{~kg} / \mathrm{m}^{3}$ and the dynamic viscosity is $\mu=0.1 \mathrm{~Pa} . \mathrm{s}\left(\right.$ at $T=20^{\circ} \mathrm{C}$ ). Size of the steel sphere, Re and St numbers are $0.0053 \mathrm{~m}, 30$ and 55, respectively. The trajectory/distance-from-wall of the particle is affected by particle-fluid and particle-wall interactions.

The particle trajectory/distance-from-wall and the interactions with fluid and wall will be investigated numerically in STAR$\mathrm{CCM}+$ using Coupled Computational Fluid Dynamics and Discrete Element Method (CFD-DEM). DEM simulations are capable of tracking individual particles' trajectories, considering various forces on the particles caused by interaction with fluid, with other particles, and with walls. Also, STARCCM+ can simulate non-spherical particles.

This work shows that the developed model predicts the trajectory/distance-from-wall of the bouncing spherical particle with a RSME equal or less than 0.1. Also, the trajectory/distance-from-wall of the polyhedral particle differs from the spherical particles, demonstrating that shape matters. Changes in the drag force due to changes in particle shape will be also investigated. Further work will apply the model findings to the study of particle filtration. 